\title{
The Periodic Solution of Fractional Oscillation Equation with Periodic Input
}

\author{
Jun-Sheng Duan ${ }^{1,2}$ \\ ${ }^{1}$ School of Sciences, Shanghai Institute of Technology, Shanghai 201418, China \\ ${ }^{2}$ School of Mathematics and Information Sciences, Zhaoqing University, Zhaoqing, Guangdong 526061, China \\ Correspondence should be addressed to Jun-Sheng Duan; duanjssdu@sina.com
}

Received 5 July 2013; Revised 14 August 2013; Accepted 15 August 2013

Academic Editor: Ming Li

Copyright (c) 2013 Jun-Sheng Duan. This is an open access article distributed under the Creative Commons Attribution License, which permits unrestricted use, distribution, and reproduction in any medium, provided the original work is properly cited.

\begin{abstract}
The periodic solution of fractional oscillation equation with periodic input is considered in this work. The fractional derivative operator is taken as ${ }_{-\infty} D_{t}^{\alpha}$, where the initial time is $-\infty$; hence, initial conditions are not needed in the model of the present fractional oscillation equation. With the input of the harmonic oscillation, the solution is derived to be a periodic function of time $t$ with the same circular frequency as the input, and the frequency of the solution is not affected by the system frequency $c$ as is affected in the integer-order case. These results are similar to the case of a damped oscillation with a periodic input in the integer-order case. Properties of the periodic solution are discussed, and the fractional resonance frequency is introduced.
\end{abstract}

\section{Introduction}

Fractional calculus has been used in the mathematical description of real problems arising in different fields of science. It covers the fields of viscoelasticity, anomalous diffusion, analysis of feedback amplifiers, capacitor theory, fractances, generalized voltage dividers, electrode-electrolyte interface models, fractional multipoles, fitting of experimental data, and so on [1-5]. Scientists and engineers became aware of the fact that the description of some phenomena is more accurate when the fractional derivative is used. In recent years, even fractional-order models of happiness [6] and love [7] have been developed, and they are claimed to give a better representation than the integer-order dynamical systems approach.

The fractional differential and integral operators have been extensively applied to the field of viscoelasticity [8]. The use of fractional calculus for the mathematical modelling of viscoelastic materials is quite natural. The main reasons for the theoretical development are the wide use of polymers in various fields of engineering.

The theorem of existence and uniqueness of solutions for fractional differential equations has been presented in $[1,2$, $9,10]$. The theory and applications of fractional differential equations are much involved [1-5, 11-17]. Fractional oscillation equations were introduced and discussed by Caputo [18], Bagley and Torvik [19], Beyer and Kempfle [20], Mainardi [21], Gorenflo and Mainardi [22], and others.

Fractional oscillators and fractional dynamical systems were investigated in [23-28]. Achar et al. [23] and Al-rabtah et al. [24] studied the response characteristics of the fractional oscillator. Li et al. [25] considered the impulse response and the stability behavior of a class of fractional oscillators. Lim et al. [26] established the relationship between fractional oscillator processes and the corresponding fractional Brownian motion processes. Lim and Teo [27] introduced a fractional oscillator process as a solution of a stochastic differential equation with two fractional orders. Li [28] proposed an approach to approximate ideal filters by using frequency responses of fractional order.

Let $f(t)$ be piecewise continuous on $(a,+\infty)$ and integrable on any subinterval $(a, t)$. Then, the Riemann-Liouville fractional integral of $f(t)$ is defined as [1-4]

$$
{ }_{a} J_{t}^{\alpha} f(t):=\int_{a}^{t} \frac{(t-s)^{\alpha-1}}{\Gamma(\alpha)} f(s) d s, \quad \alpha>0,
$$

where $\Gamma(\cdot)$ is Euler's gamma function. 
Let $f^{(n)}(t)$ be piecewise continuous on $(a,+\infty)$ and integrable on any subinterval $(a, t)$. Then, the Caputo fractional derivative of $f(t)$ of order $\alpha, n-1<\alpha<n$, is defined as [1-4]

$$
{ }_{a} D_{t}^{\alpha} f(t):={ }_{a} J_{t}^{n-\alpha} f^{(n)}(t), \quad n-1<\alpha<n, n \in \mathbb{N}^{+} .
$$

It is well-known that the fractional oscillation equation

$$
\begin{gathered}
{ }_{0} D_{t}^{\alpha} x(t)+c^{\alpha} x(t)=0, \quad 1<\alpha<2, \\
x(0)=c_{0}, \quad x^{\prime}(0)=c_{1}
\end{gathered}
$$

does not have a periodic solution $[21,22,29,30]$. The existence of periodic solutions is often a desired property in dynamical systems, constituting one of the most important research directions in the theory of dynamical systems. The existence of weighted pseudo-almost periodic solutions of fractional-order differential equations has been investigated in [31].

In this work, we consider the fractional oscillation equation with periodic input using the fractional derivative operator ${ }_{-\infty} D_{t}^{\alpha}$. We will derive a periodic solution for this equation and discuss its properties. Since we do not consider the effect of initial values, so the periodic solution can be regarded as an asymptotic steady-state solution for a fractional oscillation with initial conditions.

For a classic undamped oscillation with the periodic input

$$
\begin{gathered}
x^{\prime \prime}(t)+c^{2} x(t)=b \cos (\lambda t), \quad c>0, \quad b>0, \lambda>0, \\
x(0)=c_{0}, \quad x^{\prime}(0)=c_{1},
\end{gathered}
$$

we list their solutions as follows.

(i) If $\lambda \neq c$,

$$
x(t)=c_{0} \cos (c t)+c_{1} \frac{\sin (c t)}{c}+\frac{b(\cos (\lambda t)-\cos (c t))}{c^{2}-\lambda^{2}} .
$$

(ii) If $\lambda=c$,

$$
x(t)=c_{0} \cos (c t)+c_{1} \frac{\sin (c t)}{c}+\frac{b t \sin (c t)}{2 c} .
$$

In the next section, as a comparison we solve the fractional oscillation equation using the fractional derivative operator ${ }_{0} D_{t}^{\alpha}$ with the periodic input and initial conditions. In Section 3, we consider the fractional oscillation equation using the fractional derivative operator ${ }_{-\infty} D_{t}^{\alpha}$ with the periodic input.

We consider the periodic problem of linear fractional differential system. For nonlinear fractional differential system, the problem is more challenging and some contributions have been made. For example, Li and Ma [32] gave the linearization and stability theorems of the nonlinear fractional system.

\section{Fractional Oscillation Equation with Periodic Input and Initial Conditions}

In this section, we solve the fractional oscillation equation with the periodic input and initial conditions

$$
\begin{gathered}
{ }_{0} D_{t}^{\alpha} x(t)+c^{\alpha} x(t)=b \cos (\lambda t), \\
c>0, \quad b>0, \quad \lambda>0, \quad 1<\alpha<2, \\
x(0)=c_{0}, \quad x^{\prime}(0)=c_{1} .
\end{gathered}
$$

The Laplace transform of (7) gives

$$
s^{\alpha} \tilde{x}(s)+c^{\alpha} \tilde{x}(s)=c_{0} s^{\alpha-1}+c_{1} s^{\alpha-2}+b \mathscr{L}[\cos (\lambda t)],
$$

where $\mathscr{L}$ denotes the Laplace transform

$$
\mathscr{L}[x(t)]=\tilde{x}(s):=\int_{0}^{\infty} e^{-s t} x(t) d t .
$$

Solving for $\tilde{x}(s)$ yields

$$
\tilde{x}(s)=c_{0} \frac{s^{\alpha-1}}{c^{\alpha}+s^{\alpha}}+c_{1} \frac{s^{\alpha-2}}{c^{\alpha}+s^{\alpha}}+b \frac{1}{c^{\alpha}+s^{\alpha}} \mathscr{L}[\cos (\lambda t)] .
$$

Upon applying the inverse Laplace transform, we obtain

$$
\begin{aligned}
x(t)= & c_{0} E_{\alpha, 1}\left(-(c t)^{\alpha}\right)+c_{1} t E_{\alpha, 2}\left(-(c t)^{\alpha}\right) \\
& +b\left(t^{\alpha-1} E_{\alpha, \alpha}\left(-(c t)^{\alpha}\right)\right) * \cos (\lambda t),
\end{aligned}
$$

where $E_{\alpha, \beta}(\cdot)$ denotes the Mittag-Leffler function $[1,21,22]$

$$
E_{\alpha, \beta}(z)=\sum_{k=0}^{\infty} \frac{z^{k}}{\Gamma(\alpha k+\beta)}
$$

and $*$ denotes the Laplace convolution

$$
\begin{aligned}
& \left(t^{\alpha-1} E_{\alpha, \alpha}\left(-(c t)^{\alpha}\right)\right) * \cos (\lambda t) \\
& \quad=\int_{0}^{t} \tau^{\alpha-1} E_{\alpha, \alpha}\left(-(c \tau)^{\alpha}\right) \cos (\lambda(t-\tau)) d \tau,
\end{aligned}
$$

and where the Laplace transform formula [1]

$$
\mathscr{L}\left[t^{\alpha-\beta-1} E_{\alpha, \alpha-\beta}\left(-c t^{\alpha}\right)\right]=\frac{s^{\beta}}{c+s^{\alpha}}
$$

is used. Since

$$
\mathscr{L}[\cos (\lambda t)]=\frac{s}{\lambda^{2}+s^{2}},
$$

the convolution in (14) can also be expressed as the following inverse Laplace transform:

$$
\begin{aligned}
Y(t) & :=\left(t^{\alpha-1} E_{\alpha, \alpha}\left(-(c t)^{\alpha}\right)\right) * \cos (\lambda t) \\
& =\mathscr{L}^{-1}\left[\frac{s}{\left(c^{\alpha}+s^{\alpha}\right)\left(\lambda^{2}+s^{2}\right)}\right] .
\end{aligned}
$$




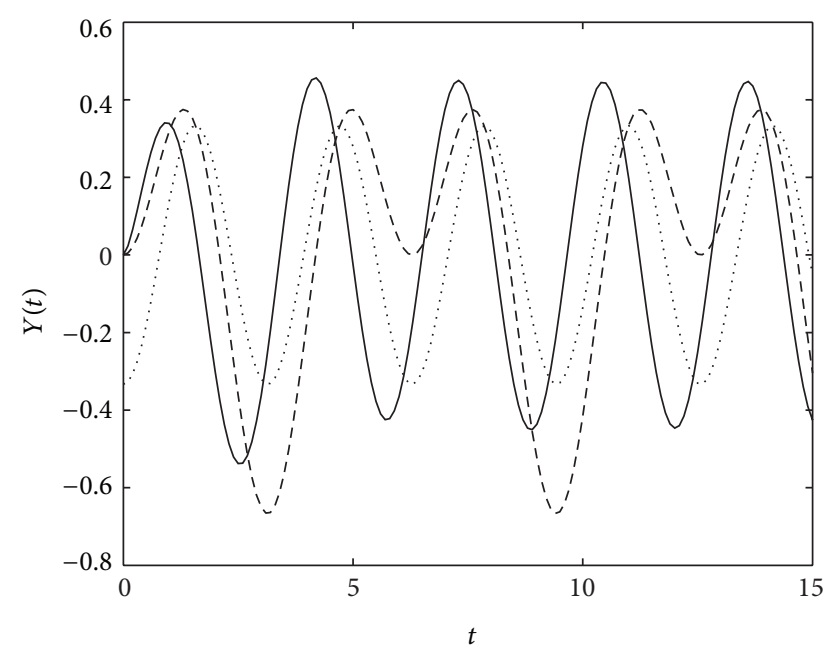

FIGURE 1: Solid line: $Y(t)$ versus $t$ on interval $0 \leq t \leq 15$ for $c=$ $1, \lambda=2$, and $\alpha=1.5$; dashed line: $(\cos (2 t)-\cos (t)) /-3$ versus $t$; doted line: $\cos (2 t) /-3$ versus $t$.

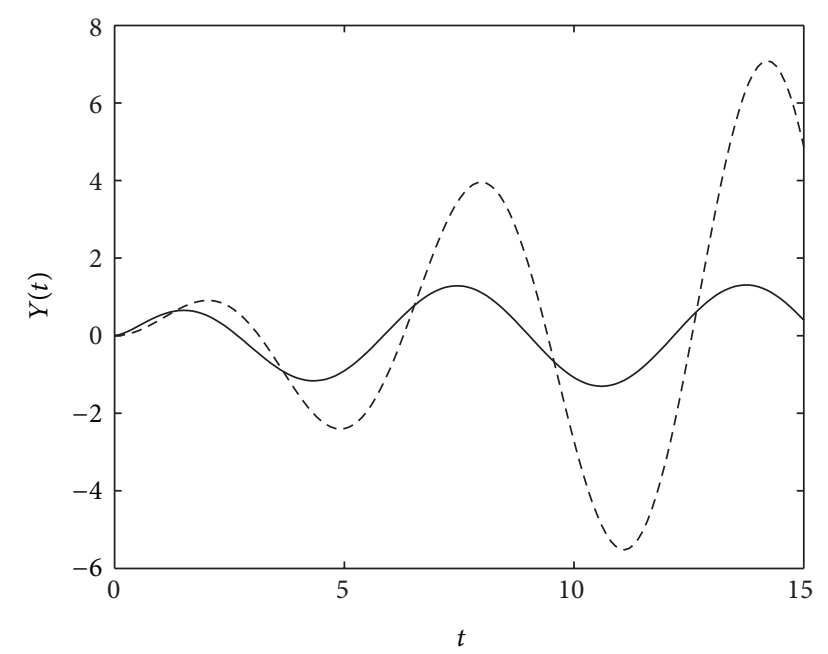

Figure 2: Solid line: $Y(t)$ versus $t$ on interval $0 \leq t \leq 15$ for $c=$ $1, \lambda=1$, and $\alpha=1.5$; dashed line: $t \sin (t) / 2$ versus $t$.

In Figure 1, the curve of $Y(t)$ versus $t$ on interval $0 \leq t \leq$ 15 for $c=1, \lambda=2$, and $\alpha=1.5$ is plotted. In order to compare with the case of $\alpha=2$ in (5), we also plot the curves of $(\cos (\lambda t)-\cos (c t)) /\left(c^{2}-\lambda^{2}\right)$ versus $t$ and $\cos (\lambda t) /\left(c^{2}-\lambda^{2}\right)$ versus $t$ for $c=1$ and $\lambda=2$ in Figure 1. We observe that with the increasing of $t$, the fractional oscillation $Y(t)$ is more relative to the function $\cos (\lambda t) /\left(c^{2}-\lambda^{2}\right)$ than to the function $(\cos (\lambda t)-\cos (c t)) /\left(c^{2}-\lambda^{2}\right)$. This means that in the fractional case, the effect of the natural frequency $c$ of the system dies out with the passage of time, which displays a damping feature and is different from the integer-order case in (5).

In Figure 2, the curve of $Y(t)$ versus $t$ on interval $0 \leq$ $t \leq 15$ for $c=1, \lambda=1$ and $\alpha=1.5$ is plotted. In order to compare with the case of $\alpha=2$ in (6), we also plot the curve of $t \sin (c t) / 2 c$ versus $t$ for $c=1$ in Figure 2 .
The Mittag-Leffler functions in (12) have the following asymptotic behaviour [1]:

$$
\begin{aligned}
E_{\alpha, 1}\left(-(c t)^{\alpha}\right) & \sim \frac{(1-\alpha) c^{-\alpha}}{\Gamma(2-\alpha)} t^{-\alpha}, \quad t \longrightarrow+\infty, \\
t E_{\alpha, 2}\left(-(c t)^{\alpha}\right) & \sim \frac{c^{-\alpha}}{\Gamma(2-\alpha)} t^{-(\alpha-1)}, \quad t \longrightarrow+\infty, \\
t^{\alpha-1} E_{\alpha, \alpha}\left(-(c t)^{\alpha}\right) & \sim \frac{\alpha(1-\alpha) c^{-2 \alpha}}{\Gamma(2-\alpha)} t^{-(\alpha+1)}, \quad t \longrightarrow+\infty .
\end{aligned}
$$

None of the three Mittag-Leffler functions in (12) are periodic. Numerical simulation displays that the convolution $Y(t)$ in (17) is not periodic, either.

If $\alpha=2$, the Mittag-Leffler functions in (12) become

$$
E_{2,1}\left(-(c t)^{2}\right)=\cos (c t), \quad t E_{2,2}\left(-(c t)^{2}\right)=\frac{\sin (c t)}{c} .
$$

In this case, calculating the convolution $\sin (c t) * \cos (\lambda t)$ for the two cases $\lambda \neq c$ and $\lambda=c$, we obtain the classical results (5) and (6) from (12).

But for the fractional case, $1<\alpha<2$, the Mittag-Leffler function $t^{\alpha-1} E_{\alpha, \alpha}\left(-(c t)^{\alpha}\right)$ in (17) approaches asymptotically $t^{-\alpha-1}$ as $t \rightarrow+\infty$, so $Y(t)$ is dominated by $\cos (\lambda t)$ as $t \rightarrow$ $+\infty$.

We note that it is possible to obtain exact periodic solutions in impulsive fractional-order dynamical systems by choosing the correct impulses at the right moments of time [29].

\section{Derivation of the Periodic Solutions for Fractional Oscillation Equation}

We consider the fractional oscillation equation using the fractional derivative operator ${ }_{-\infty} D_{t}^{\alpha}$ :

$$
\begin{array}{r}
-\infty D_{t}^{\alpha} x(t)+c^{\alpha} x(t)=b \cos (\lambda t), \\
c>0, \quad b>0, \quad \lambda>0, \quad 1<\alpha<2 .
\end{array}
$$

Equation (20) does not need to subject to initial conditions, and its solution is steady-state.

We use the following Fourier transform and its inverse:

$$
\begin{gathered}
\mathscr{F}[g(t) ; \omega]=G(\omega):=\int_{-\infty}^{\infty} g(t) e^{i \omega t} d t, \\
\mathscr{F}^{-1}[G(\omega) ; t]=g(t):=\frac{1}{2 \pi} \int_{-\infty}^{\infty} G(\omega) e^{-i \omega t} d \omega,
\end{gathered}
$$

and the Fourier transform formulas $[1,33]$

$$
\begin{gathered}
\mathscr{F}\left[{ }_{-\infty} D_{t}^{\alpha} x(t) ; \omega\right]=(-i \omega)^{\alpha} X(\omega), \\
\mathscr{F}\left[e^{i \lambda t} ; \omega\right]=2 \pi \delta(\omega+\lambda),
\end{gathered}
$$

where $\delta(\cdot)$ is the Dirac's delta function.

We rewrite the right hand side of (20) as a complex exponential function and first solve the equation

$$
{ }_{-\infty} D_{t}^{\alpha} x(t)+c^{\alpha} x(t)=b e^{i \lambda t} .
$$


The real part of the solution of (23) is the solution of (20). Applying the Fourier transform to (23) we obtain

$$
(-i \omega)^{\alpha} X(\omega)+c^{\alpha} X(\omega)=2 \pi b \delta(\omega+\lambda),
$$

from which we solve, for $X(\omega)$,

$$
X(\omega)=\frac{2 \pi b \delta(\omega+\lambda)}{c^{\alpha}+(-i \omega)^{\alpha}} .
$$

Calculating the inverse Fourier transform leads to

$$
x(t)=\mathscr{F}^{-1}\left[\frac{2 \pi b \delta(\omega+\lambda)}{c^{\alpha}+(-i \omega)^{\alpha}} ; t\right]=\frac{b e^{i \lambda t}}{c^{\alpha}+(i \lambda)^{\alpha}} .
$$

Then, we take the real part of (26) and obtain the solution of (20):

$$
\begin{aligned}
& x(t) \\
& =b \frac{\left(c^{\alpha}+\lambda^{\alpha} \cos (\alpha \pi / 2)\right) \cos (\lambda t)+\lambda^{\alpha} \sin (\alpha \pi / 2) \sin (\lambda t)}{\left(c^{\alpha}+\lambda^{\alpha} \cos (\alpha \pi / 2)\right)^{2}+\lambda^{2 \alpha} \sin ^{2}(\alpha \pi / 2)} .
\end{aligned}
$$

Obviously, (27) represents a periodic solution with the same circular frequency as the input $b \cos (\lambda t)$. Furthermore, (27) can be rewritten as the form

$$
x(t)=\frac{b \cos (\lambda t-C)}{\sqrt{\left(c^{\alpha}+\lambda^{\alpha} \cos (\alpha \pi / 2)\right)^{2}+\lambda^{2 \alpha} \sin ^{2}(\alpha \pi / 2)}},
$$

where the phase angle is

$$
C=\tan ^{-1} \frac{\lambda^{\alpha} \sin (\alpha \pi / 2)}{c^{\alpha}+\lambda^{\alpha} \cos (\alpha \pi / 2)},
$$

and the amplitude is

$$
A=A(\alpha, \lambda)=\frac{b}{\sqrt{\left(c^{\alpha}+\lambda^{\alpha} \cos (\alpha \pi / 2)\right)^{2}+\lambda^{2 \alpha} \sin ^{2}(\alpha \pi / 2)}} .
$$

The curves of $x(t)$ versus $t$ for $b=1, c=1, \lambda=1.5$, and different $\alpha$ and the curves of $x(t)$ versus $t$ for $b=1, c=1, \alpha=$ 1.5 and different $\lambda$ are plotted in Figures 3 and 4 , respectively.

The effects of the order $\alpha$ and the input circular frequency $\lambda$ on the amplitude $A$ are interesting. The curves of $A$ versus $\alpha$ for $b=1, c=1$ and, different $\lambda$ and the curves of $A$ versus $\lambda$ for $b=1, c=1$, and different $\alpha$ are plotted in Figures 5 and 6 , respectively.

Similar to a damped oscillation with a periodic input in an integer-ordered case, we observe that the curves of $A$ versus $\lambda$ have a peak value. Furthermore, the derivative of the amplitude with respective to the frequency $\lambda$ is calculated to be

$$
\frac{d A}{d \lambda}=-\frac{\alpha b \lambda^{\alpha-1}\left(\lambda^{\alpha}+c^{\alpha} \cos (\pi \alpha / 2)\right)}{\left(\lambda^{2 \alpha}+c^{2 \alpha}+2 c^{\alpha} \lambda^{\alpha} \cos (\pi \alpha / 2)\right)^{3 / 2}} .
$$

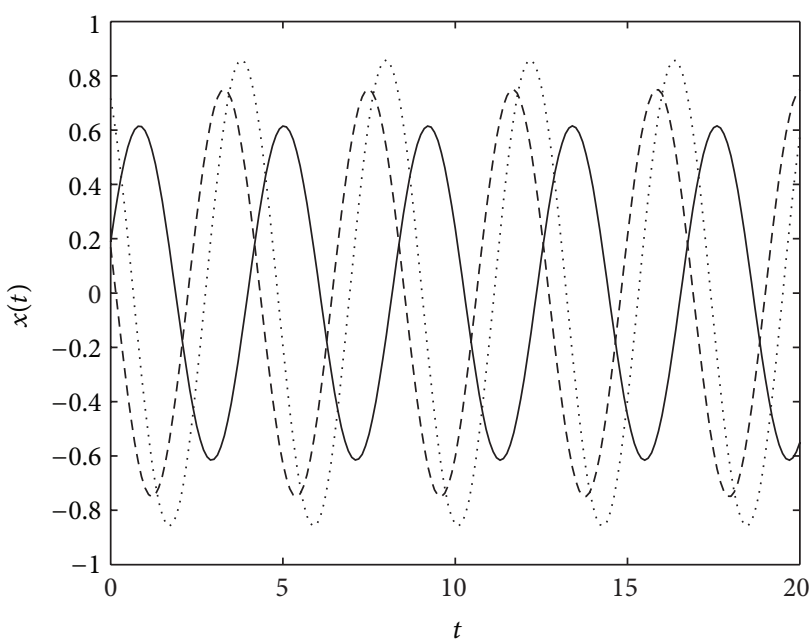

Figure 3: Curves of $x(t)$ versus $t$ for $b=1, c=1$, and $\lambda=1.5$ and for $\alpha=1.2$ (solid line), $\alpha=1.5$ (dashed line), and $\alpha=1.8$ (doted line).

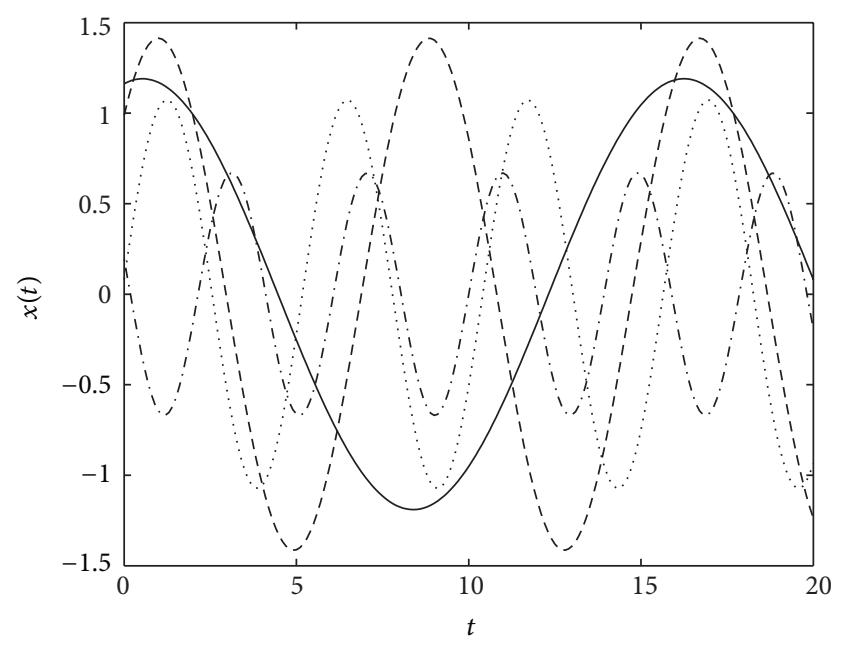

Figure 4: Curves of $x(t)$ versus $t$ for $b=1, c=1$, and $\alpha=1.5$ and for $\lambda=0.4$ (solid line), $\lambda=0.8$ (dashed line), $\lambda=1.2$ (doted line), and $\lambda=1.6$ (doted-dashed line).

Letting $d A / d \lambda=0$, we obtain

$$
\lambda^{*}=c\left(-\cos \left(\frac{\pi \alpha}{2}\right)\right)^{1 / \alpha}
$$

For each specified $\alpha, 1<\alpha<2$, the amplitude $A=$ $A(\alpha, \lambda)$ takes the maximum $A_{\max }(\alpha)$, when $\lambda=\lambda^{*}$. We call $\lambda^{*}$ the fractional resonance frequency. From (32) the resonance frequency $\lambda^{*}$ increases monotonically from 0 to $c$ with increasing of $\alpha$ from 1 to 2 . The curve of $\lambda^{*}$ versus $\alpha$ for $c=1$ is plotted in Figure 7 .

The maximum amplitude is calculated to be

$$
A_{\max }(\alpha)=A\left(\alpha, \lambda^{*}\right)=\frac{b}{c^{\alpha} \sin (\pi \alpha / 2)} .
$$




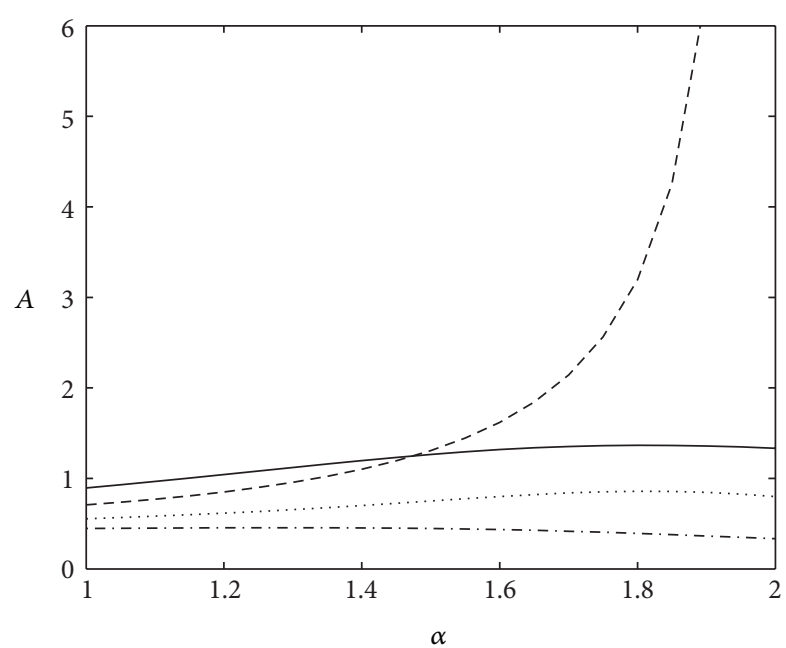

Figure 5: Curves of $A$ versus $\alpha$ for $b=1$ and $c=1$ and for $\lambda=0.5$ (solid line), $\lambda=1$ (dashed line), $\lambda=1.5$ (doted line), and $\lambda=2$ (doted-dashed line).

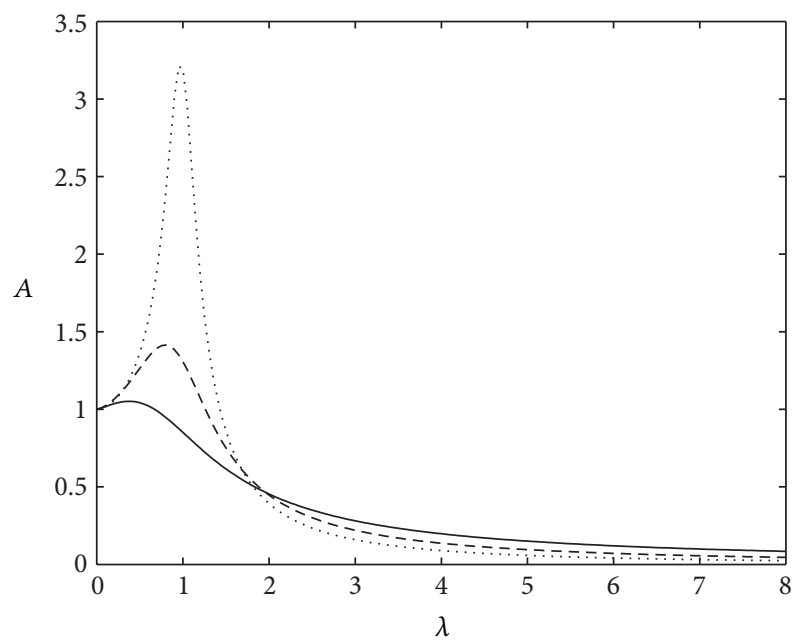

FIgURe 6: Curves of $A$ versus $\lambda$ for $b=1$ and $c=1$ and for $\alpha=1.2$ (solid line), $\alpha=1.5$ (dashed line), and $\alpha=1.8$ (doted line).

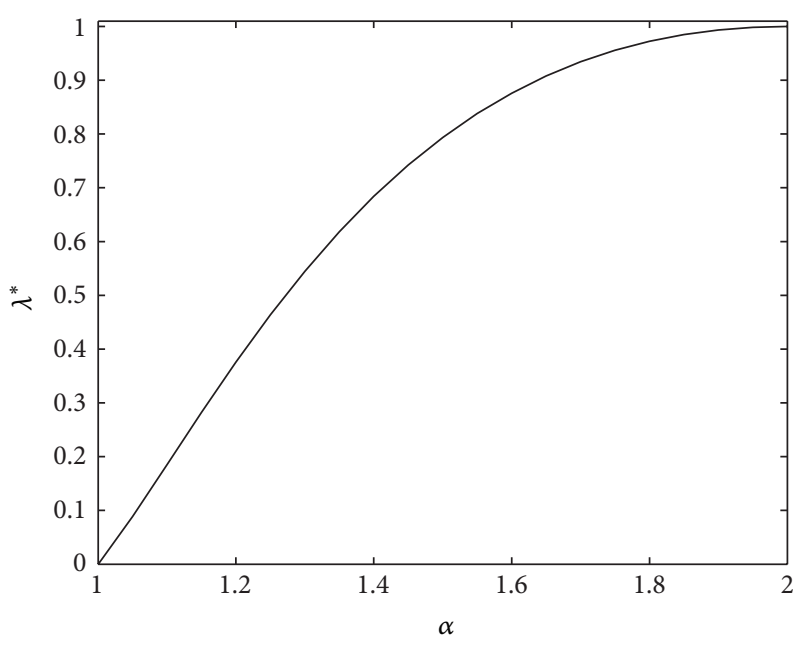

Figure 7: Curve of $\lambda^{*}$ versus $\alpha$ for $c=1$.

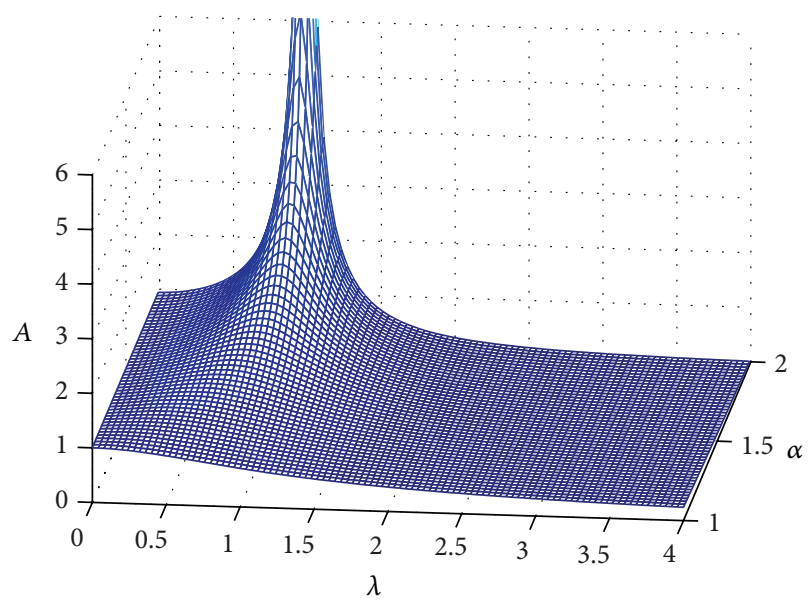

FIgURE 8: Surface of amplitude $A(\alpha, \lambda)$ for $b=c=1$.

It follows from (33) that

$$
\begin{aligned}
& A_{\max }(\alpha) \longrightarrow \frac{b}{c^{\alpha}}, \quad \alpha \longrightarrow 1^{+}, \\
& A_{\max }(\alpha) \longrightarrow+\infty, \quad \alpha \longrightarrow 2^{-} .
\end{aligned}
$$

The surface of the amplitude $A(\alpha, \lambda)$ for $b=c=1$ is shown in Figure 8.

\section{Conclusions}

The fractional oscillation equations with a harmonic periodic input are considered for the fractional derivative operators ${ }_{0} D_{t}^{\alpha}$ and ${ }_{-\infty} D_{t}^{\alpha}$, respectively. For the latter fractional oscillation equation, the periodic solution with the same circular frequency as the input function is derived. The solution is similar to the case of a damped oscillation with a periodic input in the integer-order case, and a fractional resonance frequency occurs. The frequency of the solution of the fractional oscillation equation is not affected by the system frequency $c$. The results show that the fractional oscillation equations represent the damping feature. We give a detailed analysis for the effects of the order $\alpha$ and the input circular frequency $\lambda$ on the oscillation amplitude $A$. The periodic solution can be regarded as an asymptotic steady-state solution for a fractional oscillation with initial conditions.

\section{Acknowledgment}

This work was supported by the National Natural Science Foundation of China (no. 11201308) and the Innovation Program of Shanghai Municipal Education Commission (no. 14ZZ161).

\section{References}

[1] I. Podlubny, Fractional Differential Equations, vol. 198 of Mathematics in Science and Engineering, Academic Press, San Diego, Calif, USA, 1999. 
[2] A. A. Kilbas, H. M. Srivastava, and J. J. Trujillo, Theory and Applications of Fractional Differential Equations, vol. 204 of North-Holland Mathematics Studies, Elsevier Science, Amsterdam, The Netherlands, 2006.

[3] F. Mainardi, Fractional Calculus and Waves in Linear Viscoelasticity, Imperial College Press, London, UK, 2010.

[4] D. Băleanu, K. Diethelm, E. Scalas, and J. J. Trujillo, Fractional Calculus Models and Numerical Methods, vol. 3 of Series on Complexity, Nonlinearity and Chaos, World Scientific Publishing, Hackensack, NJ, USA, 2012.

[5] J. T. Machado, V. Kiryakova, and F. Mainardi, "Recent history of fractional calculus," Communications in Nonlinear Science and Numerical Simulation, vol. 16, no. 3, pp. 1140-1153, 2011.

[6] L. Song, S. Xu, and J. Yang, "Dynamical models of happiness with fractional order," Communications in Nonlinear Science and Numerical Simulation, vol. 15, no. 3, pp. 616-628, 2010.

[7] R. $\mathrm{Gu}$ and $\mathrm{Y} . \mathrm{Xu}$, "Chaos in a fractional-order dynamical model of love and its control," in Nonlinear Mathematics for Uncertainty and Its Applications, S. Li, X. Wang, Y. Okazaki, J. Kawabe, T. Murofushi, and L. Guan, Eds., vol. 100 of Advances in Intelligent and Soft Computing, pp. 349-356, Springer, Berlin, Germany, 2011.

[8] Y. A. Rossikhin and M. V. Shitikova, "Applications of fractional calculus to dynamic problems of linear and nonlinear hereditary mechanics of solids," Applied Mechanics Reviews, vol. 50, no. 1, pp. 15-67, 1997.

[9] D. Băleanu, O. G. Mustafa, and R. P. Agarwal, "An existence result for a superlinear fractional differential equation," Applied Mathematics Letters, vol. 23, no. 9, pp. 1129-1132, 2010.

[10] D. Băleanu and O. G. Mustafa, "On the global existence of solutions to a class of fractional differential equations," Computers \& Mathematics with Applications, vol. 59, no. 5, pp. 1835-1841, 2010.

[11] J.-S. Duan, "Time- and space-fractional partial differential equations," Journal of Mathematical Physics, vol. 46, no. 1, Article ID 013504, pp. 13504-13511, 2005.

[12] V. Daftardar-Gejji and H. Jafari, "An iterative method for solving nonlinear functional equations," Journal of Mathematical Analysis and Applications, vol. 316, no. 2, pp. 753-763, 2006.

[13] G. Wu, "A fractional characteristic method for solving fractional partial differential equations," Applied Mathematics Letters, vol. 24, no. 7, pp. 1046-1050, 2011.

[14] H. Jafari, C. M. Khalique, and M. Nazari, "An algorithm for the numerical solution of nonlinear fractional-order Van der Pol oscillator equation," Mathematical and Computer Modelling, vol. 55, no. 5-6, pp. 1782-1786, 2012.

[15] H. Jafari, M. Nazari, D. Baleanu, and C. M. Khalique, "A new approach for solving a system of fractional partial differential equations," Computers \& Mathematics with Applications, vol. 66, no. 5, pp. 838-843, 2013.

[16] J.-S. Duan, T. Chaolu, and R. Rach, "Solutions of the initial value problem for nonlinear fractional ordinary differential equations by the Rach-Adomian-Meyers modified decomposition method," Applied Mathematics and Computation, vol. 218, no. 17, pp. 8370-8392, 2012.

[17] J. S. Duan, R. Rach, D. Baleanu, and A. M. Wazwaz, "A review of the Adomian decomposition method and its applications to fractional differential equations," Communications in Fractional Calculus, vol. 3, pp. 73-99, 2012.

[18] M. Caputo, "Linear models of dissipation whose Q is almost frequency independent-part II," Geophysical Journal of the Royal Astronomical Society, vol. 13, no. 5, pp. 529-539, 1967.
[19] R. L. Bagley and P. J. Torvik, "A generalized derivative model for an elastomer damper," The Shock and Vibration Bulletin, vol. 49, no. 2, pp. 135-143, 1979.

[20] H. Beyer and S. Kempfle, "Definition of physically consistent damping laws with fractional derivatives," Zeitschrift für Angewandte Mathematik und Mechanik, vol. 75, no. 8, pp. 623-635, 1995.

[21] F. Mainardi, "Fractional relaxation-oscillation and fractional diffusion-wave phenomena," Chaos, Solitons \& Fractals, vol. 7, no. 9, pp. 1461-1477, 1996.

[22] R. Gorenflo and F. Mainardi, "Fractional calculus: integral and differential equations of fractional order," in Fractals and Fractional Calculus in Continuum Mechanics, A. Carpinteri and F. Mainardi, Eds., vol. 378 of CISM Courses and Lectures, pp. 223-276, Springer, New York, NY, USA, 1997.

[23] B. N. N. Achar, J. W. Hanneken, and T. Clarke, "Response characteristics of a fractional oscillator," Physica A, vol. 309, no. 3-4, pp. 275-288, 2002.

[24] A. Al-rabtah, V. S. Ertürk, and S. Momani, "Solutions of a fractional oscillator by using differential transform method," Computers \& Mathematics with Applications, vol. 59, no. 3, pp. 1356-1362, 2010.

[25] M. Li, S. C. Lim, and S. Chen, "Exact solution of impulse response to a class of fractional oscillators and its stability," Mathematical Problems in Engineering, vol. 2011, Article ID 657839, 9 pages, 2011.

[26] S. C. Lim, L. Ming, and L. P. Teo, "Locally self-similar fractional oscillator processes," Fluctuation and Noise Letters, vol. 7, no. 2, pp. L169-L179, 2007.

[27] S. C. Lim and L. P. Teo, "The fractional oscillator process with two indices," Journal of Physics A, vol. 42, no. 6, Article ID 065208, 34 pages, 2009.

[28] M. Li, "Approximating ideal filters by systems of fractional order," Computational and Mathematical Methods in Medicine, vol. 2012, Article ID 365054, 6 pages, 2012.

[29] E. Kaslik and S. Sivasundaram, "Non-existence of periodic solutions in fractional-order dynamical systems and a remarkable difference between integer and fractional-order derivatives of periodic functions," Nonlinear Analysis: Real World Applications, vol. 13, no. 3, pp. 1489-1497, 2012.

[30] J.-S. Duan, Z. Wang, Y.-L. Liu, and X. Qiu, "Eigenvalue problems for fractional ordinary differential equations," Chaos, Solitons \& Fractals, vol. 46, pp. 46-53, 2013.

[31] R. P. Agarwal, B. de Andrade, and C. Cuevas, "Weighted pseudo-almost periodic solutions of a class of semilinear fractional differential equations," Nonlinear Analysis: Real World Applications, vol. 11, no. 5, pp. 3532-3554, 2010.

[32] C. Li and Y. Ma, "Fractional dynamical system and its linearization theorem," Nonlinear Dynamics, vol. 71, no. 4, pp. 621-633, 2013.

[33] B. Davies, Integral Transforms and Their Applications, Springer, New York, NY, USA, 3rd edition, 2001. 


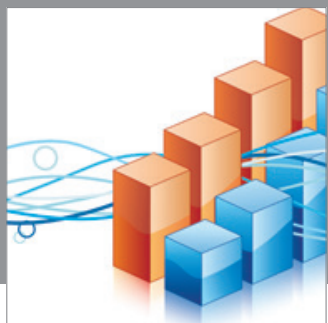

Advances in

Operations Research

mansans

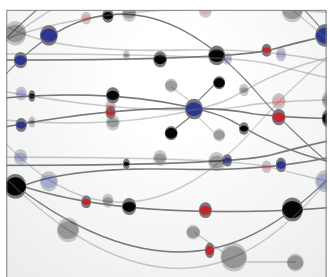

The Scientific World Journal
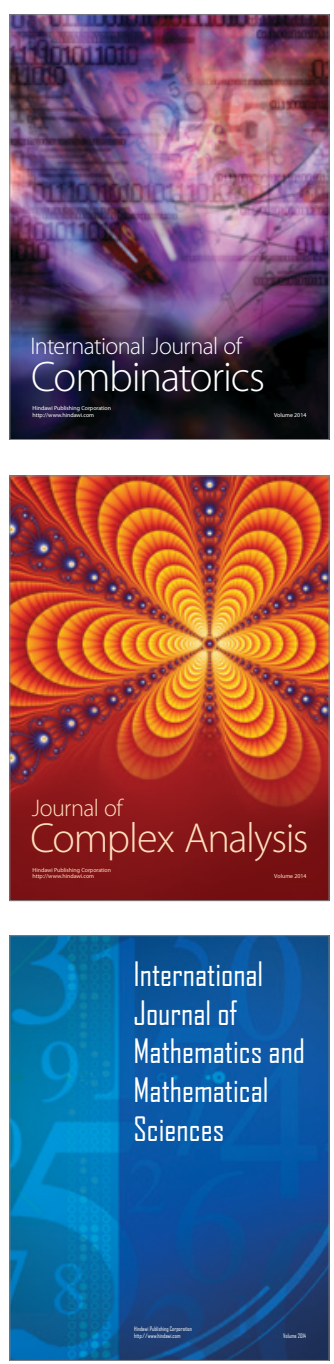
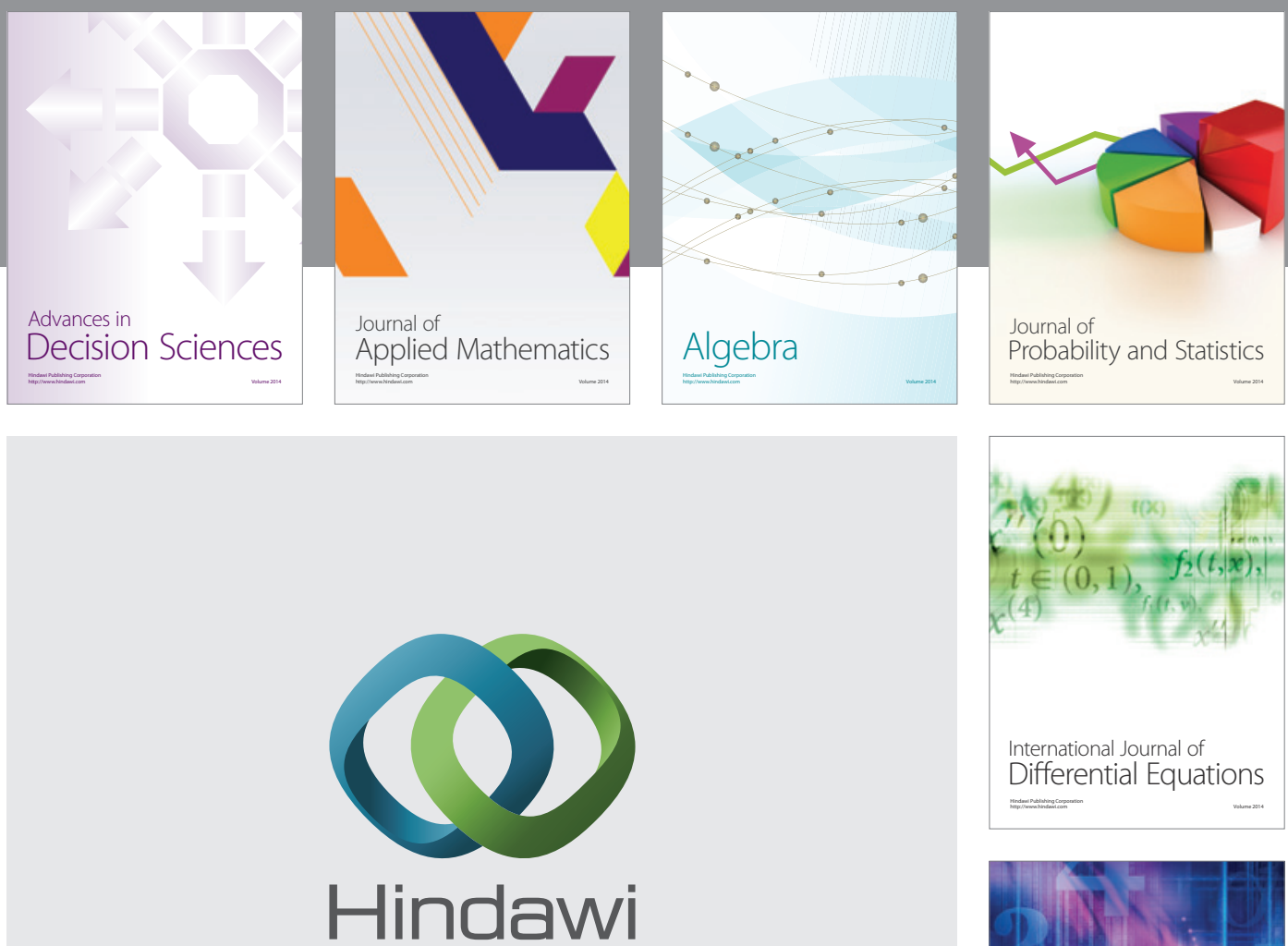

Submit your manuscripts at http://www.hindawi.com
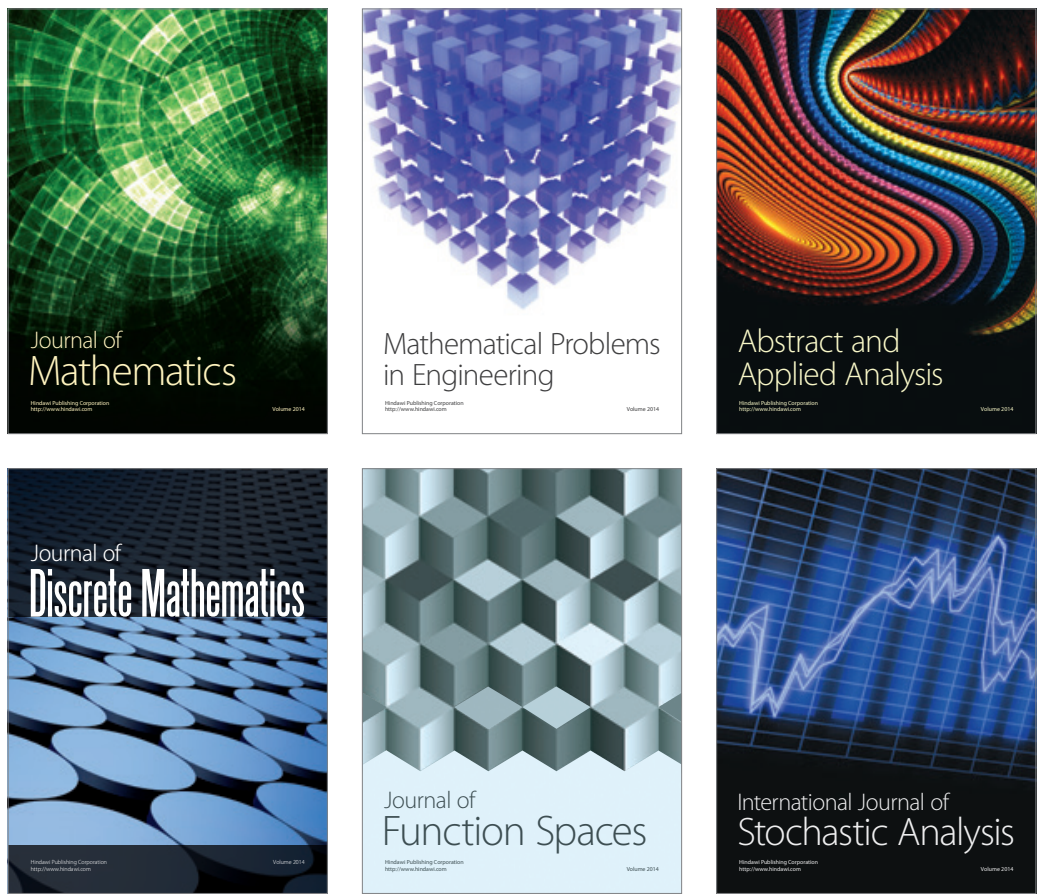

Journal of

Function Spaces

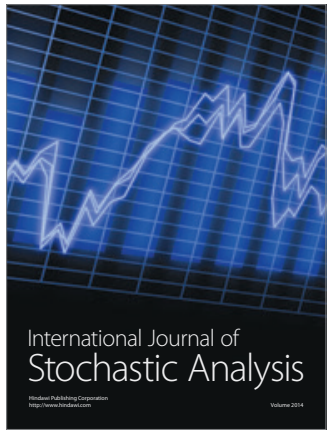

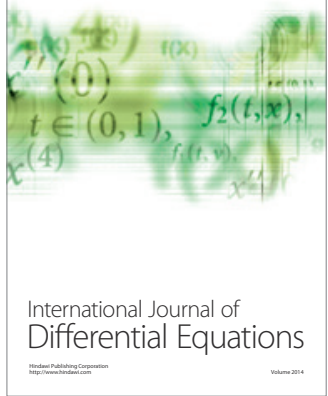
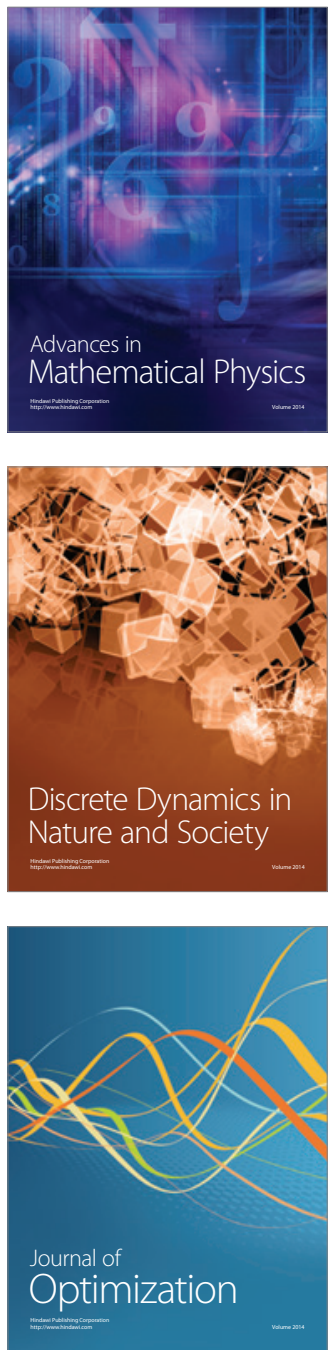